\title{
Acute Colchicine-induced Neuromyopathy in a Patient Treated with Atorvastatin and Clarithromycin
}

\author{
José M Olmos-Martínez ${ }^{1}$, Helena Molina², Cristina Salas², José M Olmos², José L Hernández² \\ ${ }^{1}$ Gastroenterology and Hepatology Department, Hospital Universitario Marqués de Valdecilla-IDIVAL, Universidad de Cantabria, Santander, Spain \\ ${ }^{2}$ Department of Internal Medicine, Hospital Universitario Marqués de Valdecilla-IDIVAL, Universidad de Cantabria, Santander, Spain
}

\section{Doi: 10.12890/2019_001066 - European Journal of Case Reports in Internal Medicine - ๑ EFIM 2019}

Received: 02/02/2019

Accepted: 05/02/2019

Published: $18 / 03 / 2019$

\begin{abstract}
How to cite this article: Olmos-Martínez JM, Molina H, Salas C, Olmos JM, Hernández JL. Acute colchicine-induced neuromyopathy in a patient treated
\end{abstract} with atorvastatin and clarithromycin. EJCRIM 2019;6: doi:10.12890/2019_001066.

Conflicts of Interests: The Authors declare that there are no competing interests.

Acknowledgements: I would like to express my gratitude to all the authors for their help gathering clinical data, manuscript redaction and review, with special thanks to Dr. Hernandez who guided us through the clinical diagnosis, management and the posterior preparation and subsequent redaction of this manuscript.

This article is licensed under a Commons Attribution Non-Commercial 4.0 License

\section{ABSTRACT}

Neuromyopathy is a rare side effect of chronic colchicine therapy, especially without renal impairment. Drugs interacting with colchicine metabolism through CYP3A4 can accelerate accumulation and toxicity. We describe a case of an interaction between atorvastatin, clarithromycin and colchicine resulting in acute neuromyopathy.

\section{LEARNING POINTS}

- Colchicine has a narrow therapeutic window, and therefore, often produces side effects.

- Special caution should be adopted if patients with renal disease and concomitant medications are given colchicine.

- Before prescribing colchicine, the clinical history, including previous medications and conditions, should be carefully considered.

\section{KEYWORDS}

Colchicine, neuromyopathy, atorvastatin, clarithromycin

\section{INTRODUCTION}

Colchicine has been synthesised for the treatment and prophylaxis of gout for centuries. It has a narrow therapeutic window, with common side effects such as gastroenteritis, blood dyscrasias, and dermatitis. Neuromuscular toxicity associated with colchicine is rare and underrecognised ${ }^{[1]}$. Although it is widely used, myopathy has not been reported frequently in the literature. When noticed, it has usually been associated with renal impairment (RI) and/or co-administration of cytochrome P450-metabolised drugs ${ }^{[2]}$. We report a patient with colchicine-induced neuromyopathy after an increased dosage due to an acute gout attack shortly after receiving clarithromycin.

\section{CASE DESCRIPTION}

A 78-year-old Caucasian man, with a history of hypertensive myocardiopathy and chronic gout, was admitted because of muscle pain and weakness in the lower extremities lasting for 15 days. One month before admission, he received intravenous ertapenem and oral clarithromycin due to orchitis. He developed acute arthritis in the right first metatarsophalangeal joint, and colchicine was increased from 1 to $4 \mathrm{mg}$ per day. His current medications included: furosemide (40 mg qd), dutasteride/tamsulosin (0.5/0.4 qd), allopurinol (300 mg qd), omeprazole (20 mg qd), paracetamol (650 mg/t.i.d.), atorvastatin (40 mg qd), and verapamil (240 mg qd). 
At admission, he complained about moderate to severe weakness starting in the legs progressing to the upper limbs, in addition to myalgia without paraesthesia or sensory loss.

Physical examination revealed gouty tophi in his left elbow and at the first right metatarsophalangeal joint and a slight bilateral hypothenar atrophy. Neurological examination did not reveal any cranial nerve palsy, back pain, bladder or bowel dysfunction. The weakness affected all limbs, mainly the lower ones, without great difference between proximal and distal muscle power. The Medical Research Council (MRC) grading for muscle power was as follows:

Trapezius (left and right): 5/5; biceps (both): 4/5; triceps (both): 4/5; right psoas: 2/5; left psoas: 3/5; quadriceps (both): 3/5; right tibialis anterior: 2/5; left tibialis anterior: 4/5; right extensor hallucis longus: 2/5; left extensor hallucis longus: $3 / 5$.

He had universal areflexia and he was unable to walk. No other relevant findings were found in the physical examination.

\section{METHODS AND PROCEDURES}

Serum blood tests revealed a highly increased CK (2660 U/L), and increased LDH (457 U/L), and thyroxine levels (2.34 mUI/ml). Aminotransferases and GGT were also altered (ALT: 1238 U/L, AST: 488 U/L, FA: 83 U/L, GGT: 354 U/L) without any other abnormal parameters. Urinary tests revealed the presence of haemoglobin (+++), without red cells in the sediment, consistent with myoglobinuria. Antinuclear, antimyeloperoxidase, antiproteinase 3, antiganglionic and antineuronal antibodies were all negative. Immunoglobulin tests revealed lower levels of serum IgG and IgM $(410 \mathrm{mg} / \mathrm{dl}$ and $17.3 \mathrm{mg} / \mathrm{dl}$, respectively), consistent with a common variable immunodeficiency. A body CT scan was normal, and a spinal MRI study only showed slight multisegmental degenerative discopathy. Nerve conduction studies revealed low amplitude motor responses and low amplitude median sensory responses. Needle electrode examination showed long duration motor unit potentials, the recruitment phenomenon and diffuse fibrillation potentials most prominently in distal muscles.

In the absence of any other reasonable aetiology, and considering that the symptoms appeared right after the increase in the colchicine dosage and the introduction of clarithromycin, a diagnosis of colchicine-induced neuromyopathy was suspected. Colchicine, atorvastatin and clarithromycin were withdrawn, leading to a slow but continuous improvement in the patient's weakness and serum CK levels. Rehabilitation therapy was started, and after a few months he was able to walk on his own again. A new electrophysiological study was performed, showing normal findings at the upper limbs and a clear improvement at the lower ones. No fibrillation potentials were detected, and motor unit potentials had normal amplitudes.

\section{DISCUSSION}

Colchicine has been used for more than 200 years. Gastrointestinal side effects are quite common and often minor, whereas neuromuscular toxicity is rare and sometimes under-recognised ${ }^{[1,2]}$. The neuromyopathy associated with colchicine is usually painless, but in rare instances it can be a painful condition, as occurred in our patient. The time course is usually subacute (1-3 months) but can be acute (<4 weeks) or chronic (>3 months ${ }^{[2]}$. The key clinical finding is proximal lower limb weakness, although distal weakness and sensory involvement have been reported ${ }^{[3]}$. Laboratory tests usually show mild to higher elevations of serum CK levels. Electrophysiological findings include myopathic motor unit potentials with features of membrane irritability as may be seen in inflammatory myopathy and a coexistent sensorimotor axonal polyneuropathy ${ }^{[6]}$. Although muscle biopsy is not always necessary, it uncovers highly specific abnormalities, revealing a vacuolar myopathy with autophagic vacuoles ${ }^{[2,5]}$. Chronic RI, hepatopathy or diuretic use have been reported to be predisposing factors for this side effect at the usual dose range ${ }^{[2,4,7]}$. Moreover, it has been widely reported that co-administration of drugs that are metabolised by the CYP450 system can induce colchicine toxicity, including myopathy ${ }^{[3,5,7,8]}$. This fact is explained by the inhibition of CYP3A4 by other drugs metabolised by the same route, leading to increased levels of colchicine despite normal dosage. Our patient was being treated with two drugs reported to interact with colchicine: clarithromycin and atorvastatin. He was on statin treatment for more than 3 years, but clarithromycin was prescribed 2 weeks before symptoms of myopathy began. Accordingly, the increased dose of colchicine in a patient receiving atorvastatin and clarithromycin at the same time could probably trigger the myoneuropathy in our case.

\section{CONCLUSIONS}

Colchicine-induced myotoxicity is a rare adverse effect. Clinicians should recognise that RI is the primary risk factor for colchicine-induced myotoxicity, and that dosage adjustment or alternative therapy may be required if the patient is receiving other P450-metabolised medication at the same time. It should be suspected in elderly patients with RI who present with subacute proximal lower extremity weakness. Diagnosis may be confirmed by electromyography or muscle biopsy. Prompt discontinuation of the medication can reverse most of the clinical weakness and laboratory abnormalities. On the other hand, combined use of macrolides and colchicine should be cautioned. 


\section{REFERENCES}

1. Kuncl RW, Duncan G, Watson D, Alderson K, Rogawski MA, Peper M. Colchicine myopathy and neuropathy. N Engl J Med 1987;316:1562-1568.

Wilbur K, Makowsky M. Colchicine myotoxicity: case reports and literature review. Pharmacotherapy 2004;24:1784-1792

3. Ghosh PS, Emslie-Smith AM, Dimberg EL. Colchicine-induced myoneuropathy mimicking polyradiculoneuropathy. J Clin Neurosci 2014;21:331-332.

4. Van der Velden W, Huussen J, Ter Laak H, de Sévaux R. Colchicine-induced neuromyopathy in a patient with chronic renal failure: the role of clarithromycin. Neth J Med 2008;66:204-206.

5. Goh IW, How CH, Tavintharan S. Cytochrome P450 drug interactions with statin therapy. Singapore Med J 2013;54:131-135.

6. Kuncl RW, Cornblath DR, Avila O, et al. Electrodiagnosis of human colchicine myoneuropathy. Muscle Nerve 1989;12:360-364.

7. Mor A, Wortmann RL, Mitnick HJ, et al. Drugs causing muscle disease. Rheum Dis Clin North Am 2011;37:219-231.

8. McKinnell J, Tayek JA. Short term treatment with clarithromycin resulting in colchicine-induced rhabdomyolysis. J Clin Rheumatol 2009;15:303-305. 